\title{
THE NAUHEIM TREATMENT.
}

By A. AINSLIE HUDSON, M.D., F.R.C.S. Ed.;

Resident Physician, St. Ann's Hill Hydropathic, Blarney.

[Read in the Section of Medicine, May 18, 1906.]

1. The Nauheim Treatment, or, as it is sometimes called, the Schott-Nauheim treatment, was, as its name implies, first practised at Nauheim, in Germany, by the brothers Theodor and August Schott, to whose researches we owe most of our present knowledge of this valuable method. The treatment, as practised at Nauheim, consists in the immersion of the patient in effervescing saline baths, with the addition of certain exercises which may be merely passive, or those known as resistive exercises-the two, baths and exercises, being employed conjointly or separately; and this treatment may or may not be combined with massage, as the case may demand.

Briefly, the composition of these baths is as follows:They are derived from certain springs at Nauheim, at temperatures varying from about $60^{\circ} \mathrm{F}$. to $90^{\circ}$ or $95^{\circ} \mathrm{F}$. They are rich in salines, of which the most abundant are sodium chloride ( 1 to 3 per cent.) and calcium chloride (.2 to .5 per cent.) and bicarbonates. They contain, moreover, free carbon dioxide, which is deposited on the surface of the patient's skin in the form of minute bubbles. The irritation produced by the bursting of these bubbles, which imparts a peculiar, prickly sensation to the skin, and the stimulating action of the saline ingredients contained in the water, are the essential factors in the production of the beneficial results of this treatment. Certain modifications of these baths are to be found at different places 
on the Continent, but their effect is in the main the same, and their variety depends upon the varying quantity of the saline materials to be found in them, and also in the presence, in addition, of certain other mineral ingredients, such as iron, sulphur, \&c., the latter being an important ingredient in the Aix la Chapelle bath.

The Physiological Explanation of the Phenomena.These, as I have pointed out, are due to the combined effects of the salines in the water and to the minute bubbles of $\mathrm{CO}_{2}$ gas, which, settling on the surface of the body, and there bursting, set up an irritation which, acting on the skin, produces a general effect through the entire nervous system, thus constituting a purely nervous phenomenon.

Another theory, and one which seems to be more satisfactory, is that of Baruch, Professor of Hydro-therapeutics in the New York Post-Graduate School. His view is supported by Woods-Hutchinson, Professor of Comparative Physiology in the University of Buffalo.

They hold that it would be impossible for the heart to propel the blood through the peripheral vessels uniless these vessels were endowed with a propulsive power, capable of furthering the onward flow of the blood contained in them, and this theory has been suggested entirely by their clinical observations.

Woods-Hutchinson has adopted an ingenious term to express this power on the part of the cutaneous vessels, when he speaks of the "skin heart" as an important factor in the human circulation.

It is granted by all physiologists that the propulsion of the blood is largely due to the elasticity of the vessels whose calibre is changed by the contractions and relaxations of the powerful muscular coat; but Woods-Hutchinson goes one better. He suggests that in all probability 
these muscle cells are possessed of far wider powers than that of merely being capable of such mechanical changes as regulating the calibre of the vessels they surround. Amongst the higher invertebrates and some of the lower vertebrates this same muscular wall, throughout the entire circulatory system, plays an extraordinary active part in regulating the circulation, there being localised aggregations of the same muscular fibres in two or three more places in the body. Even in some of the higher mammals, such as the larger hoofed animals, are to be found pulsating networks of arteries, which may be regarded as accessories to the heart. The further we go down the scale the more frequently is this found to be the case. In the wing of the bat, the fins of fishes, the web and mesentery of the frog, the rabbit's ear, and elsewhere, the vessels are known to possess the power of rhythmical contraction. The heart's action is intrinsic, and when we recall the fact that the great mesh of cutaneous vessels is capable of containing over 30 per cent. of the blood in the entire body, and that the whole of this surface is affected by the bath, it would seem that it is the stimulation of this "skin heart" that is the factor in improving the circulation. Unstriped muscle cells elsewhere in the body are possessed of the power of rythmical contraction, so why not in the walls of the blood vessels? Assuming, then, that the cutaneous vessels have the power of inherent rhythmical contraction, let us see how they might be expected to behave under certain special conditions, as during the process of a Nauheim bath. The immediate effect of the application of the water, rich in saline material, is to produce a powerful stimulation of the muscular coats of the superficial blood vessels, and this is greatly enhanced by the presence of the $\mathrm{CO}_{2}$, that unrivalled stimulant of unstriped muscle fibres, thereby improving 
the tone of the vessels; but we must, if we adhere to our premise, regard this as an active rather than a reflex condition. As to the effect on the heart of this process there is some diversity of opinion. Immediately following on the local stimulation comes a rapid reactionary dilatation, which dilatation has been usually credited with the relief of the heart by diminishing the peripheral resistance. But Winternitz, on the other hand, points out that, as in the case of the fever patient, whose flushed face denotes an already distended condition of the peripheral vessels, the general improvement caused by cold sponging is rather due to an increased resistance in the peripheral circulation, and that in the same way the frictional irritation produced by the bubbles of $\mathrm{CO}_{2}$ is responsible for the improved tone of the heart by increasing their resistance. The lymphatic and venous systems are undoubtedly stimulated likewise, with the result that stagnation is removed and an abundance of blood is supplied to the right side of the heart, in itself a stimulus for systolic contraction. The kidneys, in their turn, are flushed with an abundance of blood, as evidenced by the increased urinary secretion after the treatment, so that whatever be the precise physiological phenomena, the practical effect is unmistakable. The pulsations of the heart are greatly reduced in frequency, become more regular, and are increased in force, so that the work, that was previously almost too much for it, is performed with comparative ease and comfort, and so great is the effect of the bath that in some severe cases of dilatation the apex has been known to retract three-quarters of an inch towards its normal position in a single treatment. The blood pressure is raised, the breathing becomes regular and quiet, the functional activity of the skin and kidneys is increased, and the general comfort of the individual is improved. 
Technique of the Process.--As to the length of course required, eighteen to twenty baths, and never more than thirty, are taken in sets of three, with a day's rest between each set. However, sometimes two only are taken in succession, and sometimes four. The temperature, as a rule, should be $95^{\circ}$ to $98^{\circ} \mathrm{F}$., according to the time of year. At the commencement of the course it is advisable to limit the bath to five minutes, but the time may be lengthened daily by a few minutes, until a maximum of fifteen or twenty minutes has been reached. Two hours must have elapsed since the last meal before a bath is taken, and it should not be given if the patient is tired. After the bath the patient is wrapped in a warm sheet and dried, while seated, by the nurse. This is followed by an hour's rest in the recumbent position. The pulse is taken before and after the bath, and a trained attendant must be in charge of every case, who is capable of observing any slight change in the appearance and breathing of the patient which would call for the stopping of the bath.

The Nauheim Exercises are employed as an accessory to the bath, and, as I pointed out, these exercises may be passive or resistive. Passive movements relieve the heart; resistive movements--which can be graduated from the mere weight of the operator's hand to a much more powerful resistance quicken the circulation in the muscles brought into action, and produce a reflex condition of the cardiac muscle. The resistance must be either manual or mechanical - that is to say, by means of certain mechanical devices which take the place of the attendant's manipulations in resisting the patient's movements. At the beginning movements of the upper extremities only are employed, afterwards those of the lower extremities and trunk. A pause of half to one minute occurs between each movement, and the exercises are not given within one and 
a half hours after a full meal. The dress is loose, so as not to constrict any part of the body, and no movements are repeated on the same limb at the one sitting. The movements are performed slowly, and at a regular rate. They may be given in a reclining position, sitting, or standing, according to the requirements of the case and the condition of the patient. Should signs of exhaustion appear, such as palpitation, dyspnoa, pallor, or any other signs of distress, they must be discontinued. Speaking generally, the resistive exercises consist in the patient performing the movements of flexion, extension, adduction, abduction,and circumduction, \&c., of the limbs, and certain movements of the trunk, these movements being opposed by the attendant throughout the process. These exercises may be practised at a convenient interval after the bath each day, or at a different time of the day, and may be made to alternate with the bath, according to the individual requirements and exigencies of the case, and they should be continued throughout the course of baths. It will be seen, therefore, that a full course, as given at Nauheim, entails from a month to six weeks' treatment, and as the process has undoubtedly a tendency to general relaxation of the system, patients are advised when the course is over to go for a few weeks into the mountains to be braced up.

The Indications for Nauheim Treatment.-Speaking generally, the indications for this treatment are chronic affections of the heart, especially the passively dilated heart associated with fatty infiltration as a part of general obesity or senile changes, the weakened heart left by influenza or other acute affections, the flabby, lifeless organ associated with anæmia and neurasthenia, the heart impaired by excessive strain from running or other athletic exercises, and the heart of the patient with arterio- 
sclerosis, chronic gout, or rheumatism. In a much more restricted degree is it to be employed in valvular diseases. Now, as it is beyond the possibility of every sufferer from one of the above conditions to go to Nauheim, let us see what can be done by artificial means to procure a substitute for the natural mineral springs which shall be within the reach of everyone. The essential elements in the preparation of such a bath are a proper amount of salinity and some means of setting free in the water a certain volume of carbonic acid gas. There have been several methods of doing this suggested, but for the purposes of this description it will only be necessary to mention two. For a bath of between 40 to 50 gallons add $10 \mathrm{lbs}$. of sodium chloride, 10 ounces of calcium chloride, and, after these are thoroughly dissolved, add $1 \frac{1}{2} \mathrm{lbs}$. of bicarbonate of soda. The acid is now required to complete the process of effervescence. This may be obtained by adding to the bath, just before the patient enters it, $2 \frac{1}{2}$ lbs of hydrochloric acid, which should be thoroughly mixed with the alkaline solution, when the effervescence immediately begins. This method has its disadvantages; the effervescence is too rapid, and, consequently, does not last long. enough, and unless the acid is intimately mixed with the solution the effervescence is not uniformly distributed over the whole bath, and, moreover, there is some danger from undue irritation of the skin by the incompletely diluted acid, to say nothing of the risk to the attendant of handling the strong acid. To obviate this $I$ have adopted a method employed at Harrogate in the administration of these baths. In a glass vessel provided with a stop-cock and placed on a bracket at a convenient height above the bath is poured the hydrochloric acid diluted to the extent of 1 in 6. From the stop-cock is dependent a tube of fine calibre, the lower end of which is inserted into another 
tube that encircles the bath at a distance of about six inches from the bottom, in which are arranged minute perforations at intervals, pointing inwards - that is, towards the bather. On turning the stop-cock the acid descends the vertical tube, and on entering the horizontal tube on the inside of the bath is distributed in minute jets throughout the whole bath. By this means the effervescence is rendered uniform, and the supply of acid is under control. A simpler method than the above, and one which fulfils every requirement, is that devised by Ernest Sandow, of Homburg, who supplies the necessary ingredients through his agents in London for any kind of carbonated bath (plain, brine, sulphur, or chalybeate). The quantity of carbonic acid gas liberated during the process is 3,000 to 4,000 cc.m. to every gallon of water used. Baths prepared in this way equal in their percentage of free carbonic acid the springs of Nauheim or Kissingen, Schwalbach, \&c., and by the above means an intensified local action may be obtained to the back, abdomen, or even to vagina or uterus by means of a speculum. The ingredients used in this method are in a solid and compact form, easily portable and easy of application. It is only necessary to dissolve the required amount of the alkali in the bath before the patient enters it and then to place the acid tablets on the bottom of the bath, close to the patient's body, when the evolution of the carbonic acid gas will at once begin. Should an intensified action be desired on any given part of the body drop the contents. of the alkali and acid parcels on the same spot after entering the bath, when a brisk evolution of carbonic acid will begin at once. Let me here emphasise the importance of having a skilled attendant to administer the bath, who is capable of appreciating the possible risks involved, and who can bring to 
bear upon the treatment a reasonable intelligence and solicitude for the well-being of the patient.

Before leaving the subject I would like, in conclusion, to take the opportunity of saying how strongly I deprecate the prevailing idea generally inherent in the patients themselves, that any form of hydropathic treatment, undertaken, it is understood, as often as not as a last resource in the course of some intractable chronic ailment, is to act like a charm, and that they can expect to be cured in a couple of weeks-the average time they generally devote to a "cure"-of a disability that they have, perhaps, taken ten or even twenty years to acquire and thoroughly assimilate. It is just this class of case that brings disgrace on what, in many instances, if properly and systematically undertaken, with due regard to the length of course necessary, would prove of inestimable value to a large number of sufferers. 